\title{
FS3 Framework SIGMA 3 - A complete framework \& toolkit for J2EE applications development
}

\author{
José Luis Poy ${ }^{1}$, Jordi Cuní ${ }^{2}$, Juan José Fuentes ${ }^{3}$ \\ ${ }^{1}$ SIGMA Gestión Universitaria, Alcalde Barnils 64-68 08174 Sant Cugat Barcelona, \\ jose.luis.poy@sigmaaie.org \\ ${ }^{2}$ SIGMA Gestión Universitaria, Alcalde Barnils 64-68 08174 Sant Cugat Barcelona, \\ jordi.cuni@sigmaaie.org \\ ${ }^{3}$ SIGMA Gestión Universitaria, Alcalde Barnils 64-68 08174 Sant Cugat Barcelona, \\ juanjose.fuentes@sigmaaie.org
}

Keywords

Framework, toolkit, development, J2EE, SOA.

\section{ABSTRACT}

Since 1998 SIGMA opted for development in Java, creating its own framework on which to run its business logic. Since then, SIGMA has refined and updated the framework to the latest technologies, now providing the third generation of its suite of tools, called FS3 Framework SIGMA 3, an enterprise class middleware suite of integrated features that allows developers to build high quality business applications in a short period of time.

Technology and Innovation are the backbone of the services and solutions provided, based on a highly open source development and deployment platform for J2EE5 certified application servers compliant on a multi-tier and high performance proven open architecture. Internationalization is one of SIGMA's top priorities, as well. For years SIGMA has established relationships with other European universities consortiums. Lately SIGMA has open new strategic areas of interest such as SaaS, BI, eLearning and Mobile. SIGMA focuses the development and support of two main suites of solutions VEGA Student Information System and ARGOS Research Information System.

FS3 Framework SIGMA 3 - A complete framework \& toolkit for J2EE applications development SIGMA is engaged in the development of management applications for academic life cycle, teaching and research for users of university faculties and departments, and services for teachers and students.

Since 1998 SIGMA opted for development in Java, creating its own framework on which to run its business logic. Since then, SIGMA has refined and updated the framework to the latest technologies, now providing the third generation of its suite of tools, called FS3 Framework SIGMA 3, an enterprise class middleware suite of integrated features that allows developers to build high quality business applications in a short period of time.

The framework is designed to create web applications for a three-tier architecture (web server, J2EE application server and JDBC connectivity to database). FS3 consists of four elements, the SDK itself, a service control package for security, user access management, traceability, a SOA bus to connect web services and a set of high-level tools to complete the typical needs of a multi-user application such as planning processes, data extraction, graphical report, system and applications logs analysis and so on.

Deploy FS3 applications on a cloud environment allow SIGMA to offer those applications in a SaaS model. The framework makes it possible that administrating just one production environment for the application server the same application is able to run once for multiple customers, making the administration tasks easier and reducing costs. 
FS3 Elements

- FS3 SDK

- FS3 SDK is built with Hibernate (persistence tier), IceFaces (presentation tier) generating JSP's and HTML with CSS capabilities, Spring BPM and a wide range of homemade software patterns that are result of the SIGMA's experience for usability in higher education software design (controller tier).

- FS3 integrate a customizable and configurable View Engine user interface with a clean and universal look and feel for all the features of the developed applications. This improved look and feel can be suitable for any customer and produces different outputs for laptop, tablet or smartphone.

- Providing adapted CSSs to CSS3 the View Engine output becomes a multi-device screen with a responsive web design.

- FS3 Service Control

- The Service Control package offers identification and authentication using a standard CAS protocol for single sign on, the management of the users' access to functions and data, a service manager module to control the state and the number of users allowed in each service, the generation of traces of use for further analysis of the impact on applications, etc.

- FS3 SOA BUS

- SIGMA has designed a standard multipurpose Web service that decouples the business logic of the need to interact with the system data. One of the main uses of the bus is to encapsulate queries to the database as a basis for data extraction and return XML files able to be transformed into multiple output formats using the EXPLODAT tool.

- FS3 HLT (high-level toolkit) provides a series of high-level applications to complete the SDK

- EXDAT (Data Extraction) is a multipurpose tool capable of configuring data extractions from the user, identifying patterns, selections, filters and sorting. The queries are encapsulated in XML files that are easy to implement and run through the SOA bus. The result is also transferred using XML files to the stage to output formatting in Excel or PDF, or be consumed by other applications such as FS3iD (Interactive Dashboard) or Mobile Apps.

- iDashboard is a graphical reporting tool based on HTML5 portlets using JPlot \& JQuery technology, where each portlet is a graphical representation of a defined data set generated by the SOA bus.

- CRONOS is a process scheduler that centralizes user requests to run in interactive or batch mode. The scheduler engine is the widely used open source Quartz software. The advantage of centralizing processes and reports is that they benefit from a standard pattern-based execution.

FS3 is a set of tools that greatly facilitates last areas of development and maintenance of web applications in Java and ensuring the security and performance of such applications in critical environments in real time.

\section{AUTHORS}

Jose Luis Poy, CTO Chief Technology \& Innovation Officer

MBA at Universitat Politècnica de Catalunya (2005 - 2006),

Postgraduate in Information Systems Manager at Universitat Politècnica de Catalunya (2004 - 2005),

Postgraduate in Production Manager at Institut Català de Tecnologia (2003 - 2003),

Computer and Software Engineer at Universitat Autònoma de Barcelona (1985 - 1991).

He works for SIGMA since 1994, covering different technical and managerial roles. He has been the COO Chief Operations Officer for the SIGMA's 2008-2012 Transformation Plan, managing a 100 people Software Engineering team, with activity areas in Product, Service and Market Strategy, Annual Budget for incomes and costs, Organization, Quality and Methodology, Internationalization, Full Product Life Cycle (PreSales, Execution,PostSales (Project, Product, Service), Project and Resources Management, Software Factory, J2EE Platform, Out of The Box Implantation \& SaaS, Customer Service continuous improvement, Help Desk \& SLA). As CTO, his main current activity is related to 
innovation and technology, with special interest areas as Mobile Apps, BI platforms, eLearning platforms and Cloud services. He is a member of RS3G, group in which works to facilitate the European Student Mobility, and he is also a member of EuroCRIS, organization in which deals with Research Information Systems standards.

Jordi Cuní, Software Architecture Manager

Computer Science degree at Universitat Oberta de Catalunya (2006 - 2012),

Computer and Software Engineer at Universitat Autònoma de Barcelona (1997 - 2000).

He works for SIGMA since 2000, being the current Architecture and Software Quality Assurance area manager. His role focuses mainly on defining the strategy for the FS3 SIGMA framework and software patterns development in order to increase productivity, defining the development and test methodology and establishing the software development tools for the whole company. Last but not least, he is a key member of the team for the technical and performance support to costumers. Previously, he had been a project manager for 5 years, achieving the necessary experience for the implementation of a complete framework toolkit.

Juan Jose Fuentes, Middleware \& Systems Manager

Postgraduate in Corporative Networks Design (2005 - 2005),

Microsoft Certified Systems Engineer - MCSE (2002 - 2003),

Technical Computer and Software Engineer at Universitat Autònoma de Barcelona (1998 - 2001).

He works for SIGMA since 2001, currently leading the Middleware \& Systems area. His role covers a wide range of activities from designing strategy plans for backups, recovery, monitoring and storage to defining and carrying out the design of the CPD provisioning. He maintains and upgrades the backend resources of SIGMA. He gives advice to the company customers in their hardware infrastructures. He took part in the strategic migrations projects such as database migration (Ingres to Oracle), and middleware migration ( Iplanet - Glassfish - Weblogic). In the last year his main effort has been focused on achieving a mutliclient solution in order to minimize the maintenance cost of the SaaS service that SIGMA delivers to their customers. He is currently focused on building the SIGMA Higher Education SaaS in a cloud environment. 